OPEN ACCESS

Edited by:

Patrick Kwan,

Monash University, Australia

Reviewed by:

Sonja Hochmeister,

Medical University of Graz, Austria

Adam Strzelczyk

Universitätsklinikum Frankfurt,

Germany

${ }^{*}$ Correspondence:

Meng-Han Tsai

menghan@cgmh.org.tw

Specialty section

This article was submitted to

Multiple Sclerosis and

Neuroimmunology,

a section of the journal

Frontiers in Neurology

Received: 11 July 2018 Accepted: 09 January 2019

Published: 13 February 2019

Citation:

Lin C-H, LU Y-T, Ho C-J, Shih F-Y and Tsai M-H (2019) The Different Clinical Features Between Autoimmune and Infectious Status Epilepticus.

Front. Neurol. 10:25.

doi: 10.3389/fneur.2019.00025

\section{The Different Clinical Features Between Autoimmune and Infectious Status Epilepticus}

\author{
Chih-Hsiang Lin ${ }^{1}$, Yan-Ting Lu ${ }^{1}$, Chen-Jui Ho ${ }^{1}$, Fu-Yuan Shih ${ }^{2}$ and Meng-Han Tsai ${ }^{1 *}$ \\ ${ }^{1}$ Department of Neurology, Kaohsiung Chang Gung Memorial Hospital, College of Medicine, Chang Gung University, \\ Kaohsiung, Taiwan, ${ }^{2}$ Department of Neurosurgery, Kaohsiung Chang Gung Memorial Hospital, College of Medicine, Chang \\ Gung University, Kaohsiung, Taiwan
}

Objective: The prognosis of status epilepticus (SE) is highly related to the underlying etiology. Inflammation of the central nervous system (CNS), including infection and autoimmune encephalitis, is one of the treatable conditions causing SE. The initial presentation of infectious and autoimmune CNS disorders can be quite similar, which may be difficult to differentiate at the beginning. However, treatment for these entities can be quite different. In this study, we aim to identify the differences in clinical features among patients with infectious and autoimmune SE, which could help the clinicians to select initial investigation and ensuing therapies that may improve overall outcomes.

Methods: This was a retrospective study that included 501 patients with SE within a period of 10.5-years. Patients with inflammatory etiology were collected and separated into infectious and autoimmune SE. The symptoms at onset, SE semiology, status epilepticus severity score, and END-IT score at admission, treatment for SE, and outcome (modified Rankin Scale) on discharge and last follow-up were recorded. Data on the first cerebrospinal fluid, electroencephalography, and magnetic resonance imaging were also collected.

Results: Forty-six (9.2\%) of the 501 patients had SE with inflammatory etiology. Twenty-five (5\%) patients were autoimmune SE and 21 (4.2\%) were infectious SE. Patients with autoimmune SE have younger age and female predominance. As for clinical presentations, psychosis, non-convulsive SE, and super refractory SE were more common in patients with autoimmune SE. Nevertheless, the prognosis showed no difference between the two groups.

Conclusion: The different initial clinical presentations and patient characteristics may provide some clues about the underlying etiology of SE. When inflammatory etiology is suspected in patients with SE, younger age, female sex, psychosis, non-convulsive SE, and super refractory SE are clinical features that suggest an autoimmune etiology.

Keywords: status epilepticus, inflammatory, autoimmune, infection, autoantibody 


\section{INTRODUCTION}

Status epilepticus (SE) is a neurological emergency associated with significant morbidity and mortality that usually requires admission to an intensive care unit (1-3). The goal when treating SE is to terminate the clinical and electrographic seizure activities as soon as possible (4). Even though antiepileptic drugs (AEDs) can be used to control seizures (5), the prognosis of SE is highly related to age and the underlying etiology (6-8). To further improve outcomes, targeted management of the underlying causes may be required $(4,9)$.

Brain inflammation can also cause SE $(10,11)$, including central nervous system (CNS) infections and autoimmune encephalitis (12). These conditions can be treated and may result in significantly different outcomes (13-15). Altered mental status is the most common initial presentation of inflammatory SE (12). However, it is an ambiguous sign that provides little information on the underlying etiology. Currently available investigations could help in initial differential diagnosis but have some limitations. Laboratory tests such as bacterial or viral culture, polymerase chain reaction (PCR) for specific pathogens, or autoantibody testing may not be immediately available $(13,16)$ and the results may take a few days or weeks to return. Cerebrospinal fluid (CSF) studies are useful to confirm the diagnose of bacterial infections, but are less effective in distinguishing between viral infections and autoimmune processes $(13,17)$. Magnetic resonance imaging (MRI) can provide evidence of CNS inflammation, but not the underlying cause of the inflammation (18). Electroencephalography (EEG) may sometimes show patterns that suggest a specific diagnosis, such as extreme delta brush in patients with anti-N-methyl-Daspartate (NMDA) receptor encephalitis, but the findings are mostly non-specific (19).

Only two studies have specifically addressed the differences between infectious and autoimmune etiology. Spatola et al. were the first to report that patients with an infectious etiology were older in age and had a more severe clinical presentation at first encounter (20). Subsequently, Shin et al. found that patients with an autoimmune etiology were younger (11). Herein, we retrospectively reviewed our patients with SE and an inflammatory etiology over a 10.5-year period. We aimed to identify the presenting factors that may assist clinicians in differentiating the two entities earlier, which may lead to faster targeted treatment and better patient outcomes.

\section{MATERIALS AND METHODS}

\section{Study Design}

We retrospectively reviewed the medical records of all patients with SE admitted to the Neurological Intensive Care Unit at Kaohsiung Chang Gung Memorial Hospital between January 2006 and July 2016. This study was approved by the Chang Gung Medical Foundation Institutional Review Board.

\section{Definitions and Criteria}

SE was defined as $5 \mathrm{~min}$ or more of continuous clinical and/or electrographic seizure activity or recurrent seizure activity without recovery (returning to baseline) between seizures (21). Refractory SE was defined as SE not responded to first-line therapy (benzodiazepine) or second-line therapy and requiring general anesthesia (22). Super refractory SE was defined as SE continues $24 \mathrm{~h}$ or more after the onset of anesthesia, including those cases in which the SE recurs on the reduction or withdrawal of anesthesia (22). The semiology and etiology of SE were classified according to the International League Against Epilepsy Task Force report (23).

Inflammatory SE was defined as SE due to acute inflammation of the brain parenchyma, with or without the involvement of the meninges (12), and further divided into SE due to CNS infection and autoimmune SE. Autoimmune SE included autoimmune encephalitis and systemic autoimmune disorders causing SE (23). Patients with an identified etiology for SE such as cerebrovascular disease, intracranial tumor, head trauma, metabolic disturbance, alcohol-related, AED withdrawal, neurodegenerative disease, mitochondrial disease, and medically refractory epilepsy were excluded. Patients with an unknown etiology and those without CSF data were also excluded from this study.

Autoimmune SE was defined as suggested by previous experts' consensus (16):

1. Subacute onset (rapid progression of fewer than 3 months) of working memory deficits (short-term memory loss), altered mental status, or psychiatric symptoms.

2. At least one of the followings:

- New focal CNS findings

- Seizures not explained by a previously known seizure disorder

- CSF pleocytosis (white blood cell count of more than five cells per mm3)

- MRI features suggestive of encephalitis

\section{Reasonable exclusion of alternative causes}

SE patients who had positive neuronal surface auto-antibodies testing (EUROIMMUN, Autoimmune Encephalitis Mosaic 6 assay, Germany) in serum or CSF were also considered as autoimmune SE.

Infectious SE was diagnosed if microbiologic studies demonstrated an infectious agent. Those without evidence of microbiologic studies would have to fulfill one of the underlying criteria (20): (1) fever $>38.5^{\circ} \mathrm{C}$, (2) increased white blood cell count or C-reactive protein, (3) findings highly suggestive of a bacterial infection, such as turbid CSF, neutrophilic pleocytosis, or low CSF to serum glucose ratio $(<0.5)$, or $(4)$ clinical picture suggestive of a viral origin plus lymphocytic pleocytosis on CSF study with positive PCR result or serology test shows a 4-fold increase of viral antibodies 3 weeks after the onset of illness (24).

Clinical information was recorded using a standardized evaluation form, including the symptoms at onset, SE semiology and classification, status epilepticus severity score (STESS) (25) and the END-IT score (26) at admission, treatment for SE, and outcome at discharge and last follow-up. A STESS score $\geq 3$ (25) or an END-IT score $\geq 3$ (26) suggested a poor outcome. Data on the first acquired CSF, EEG, and MRI studies were collected. The EEG was described according to 


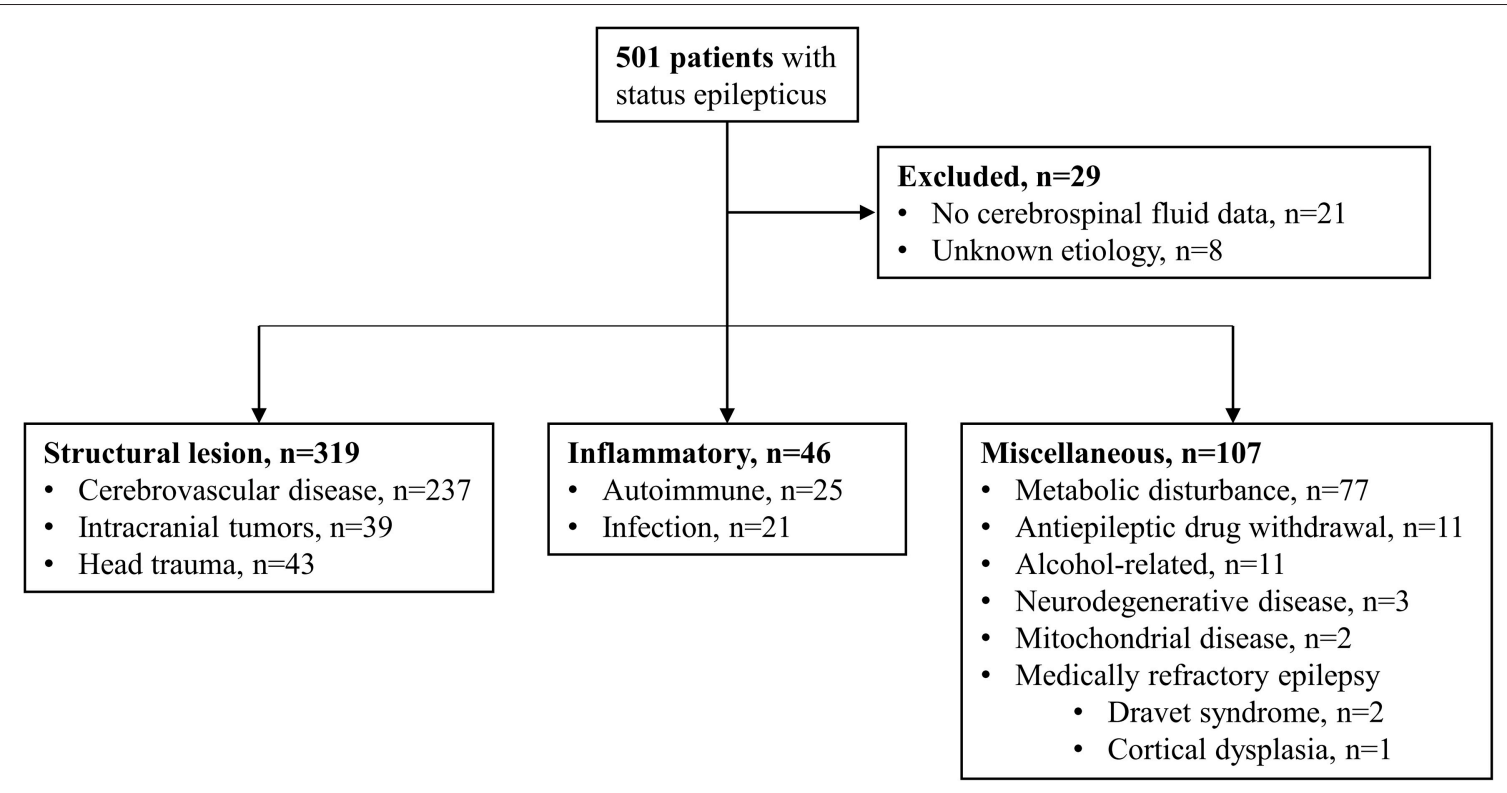

FIGURE 1 | Study population and etiologies of status epilepticus.

the 2012 American Clinical Neurophysiology Society's (ACNS) Standardized Critical Care EEG Terminology (27), which we categorized into background slowing activity, sporadic epileptiform discharge, periodic discharge, and electrographic seizures (11). MRI findings including the location and symmetry of signal changes on fluid-attenuated inversion recovery (FLAIR) and diffusion-weighted imaging (DWI) were recorded (10). Clinical outcomes at discharge and the last follow-up were graded using the modified Rankin Scale (mRS). A good outcome was defined as an $\mathrm{mRS}$ score $<3$ and a poor outcome was defined as an $\mathrm{mRS}$ score $\geqq 3$.

\section{Statistical Analysis}

Statistical analyses were performed using the IBM SPSS Statistics for Windows (version 22; IBM Corp., Armonk, NY, United States). To compare demographic data between infectious and autoimmune groups, categorical variables were assessed using Chi-square or Fisher exact tests, and continuous variables were compared using the Mann-Whitney $U$-test. $p<0.05$ was considered as statistically significant.

\section{RESULTS}

During the 10.5-year study period (January 2006-June 2016), 501 patients with SE were reviewed, of whom 46 (9.2\%) had an inflammatory etiology, including 25 females (54.3\%) and 21 males (45.7\%). Of the excluded patients, 237 had cerebrovascular disease, 77 had metabolic disturbances, 43 had head trauma, 39 had intracranial tumors, 11 had AED withdrawal, 11 had alcohol-related SE, three had neurodegenerative diseases, two had mitochondrial diseases, and three had medically refractory epilepsy. Of the three patients with medically refractory epilepsy, two had Dravet syndrome and one had focal cortical dysplasia.
Patients without CSF data $(n=21)$ and those with an unknown etiology $(n=8)$ were also excluded (Figure 1).

The clinical characteristics of the 46 patients with inflammatory SE are presented in Table 1. Among the 46 patients, 25 (54.3\%) had autoimmune SE, and 21 (45.7\%) had infectious SE. In the patients with autoimmune SE, five were related to anti-NMDA receptor encephalitis, four were related to Hashimoto encephalopathy, one was related to CNS lupus, one was related to anti-collapsin response mediator protein 5 encephalitis, and 14 were diagnosed according to the criteria of autoimmune encephalitis (16). Of these 14 patients, five had received cell-based anti-neuronal antibody assays with negative results. The remaining nine patients did not receive anti-neuronal auto-antibody tests as the test was not available at the time of diagnosis. With regards to the patients with infectious SE, six had bacterial infections, 12 had viral infections, two had cryptococcal meningitis, and one had Creutzfeldt-Jakob disease.

The clinical features of infectious and autoimmune SE are compared in Table 2. The median age at onset of the patients with autoimmune SE was younger than that of the patients with infectious SE ( 32 vs. 56, $p=0.015$ ), and more of the patients with autoimmune SE were female compared to those with infectious SE ( 68.0 vs. $38.1 \%, p=0.043)$. The initial presentation of both groups was similar, including the STESS and END-IT score at admission, onset symptoms, and latency of seizures after the initial symptoms. Psychosis was the presenting symptom only in the autoimmune SE group ( 24.0 vs. $0.0 \%, p=0.025)$ and non-convulsive SE was more prevalent among the patients with autoimmune SE compared to those with infectious SE (32.0 vs. $4.8 \%, p=0.027)$. Refractory SE occurred more commonly in the autoimmune SE than in the infectious SE group, but the difference was not statistically significant $(88.0$ vs. $66.7 \%$, $p=0.081)$. Super refractory SE was more common in the 
autoimmune SE group than in the infectious SE group (41.3 vs. $19.0 \%, p=0.007$ ). The number of AEDs used was similar between both groups, but the use of general anesthesia was more common in the autoimmune SE group than in the infectious SE group ( 64.0 vs. $23.8 \%, p=0.006$ ). However, the duration of admission or ICU stay, mRS score at discharge, and mortality rate during admission were similar between the two groups. The sensitivity and specificity for STESS to predict the outcome at discharge were 70.6 and $44.8 \%$, respectively, compared to 68.8 and $45.5 \%$ at last follow-up. The sensitivity and specificity for the END-IT score to predict the outcome at discharge were 9.4 and $100.0 \%$, respectively, compared to 21.4 and $100.0 \%$ at last follow-up.

The results of CSF and EEG are presented in Table 3 and the MRI findings are summarized in Table 4. Patients with infectious SE had a higher median CSF protein level $(93.0 \mathrm{mg} / \mathrm{dL}$ vs. $34.8 \mathrm{mg} / \mathrm{dL}, p=0.014)$, higher median white blood cell count (20 vs. 3 cell $/ \mathrm{mm}^{3}, p=0.011$ ), higher percentage of neutrophilic predominance (52.9 vs. $15.4 \%, p=0.034$ ), and higher percentage of low CSF/blood glucose ratio (56.3 vs. $24.0 \%$, $p=0.036)$ compared to the patients with autoimmune SE, who had a higher percentage of lymphocytic predominance ( 84.6 vs. $47.1 \%, p=0.034$ ). There was no significant difference in IgG index between the two groups. The autoimmune SE group tended to have a higher rate of background slowing activity in the first EEG, but the difference between autoimmune and infection was not statistically different (56.0 vs. $28.6 \%$, $p=0.081$ ). The presence of sporadic epileptiform discharge, periodic discharge, or electrographic seizure was similar among the two groups in the first EEG study. With regards to the first MRI findings, an abnormal FLAIR signal was observed in 11 patients with autoimmune SE and eight patients with infectious SE. A restricted diffusion signal on DWI was found in 14 patients with autoimmune SE and 10 patients with infectious SE. However, there were no significant differences in abnormalities in the FLAIR and DWI signals between the two groups. Detailed descriptions of the locations of the abnormal signals on FLAIR and DWI are presented in Table 4.

\section{DISCUSSION}

Inflammatory SE is a previously under-recognized subgroup of SE. In the current study, 9.2\% of all cases of SE were related to an inflammatory etiology, which is in accordance with previous studies (range from 6 to $12.8 \%)(11,20)$. Inflammatory SE has two main etiologies, infectious, and autoimmune SE, which is at times difficult to differentiate at the initial presentation. We found that younger age, female sex, the presence of psychosis, nonconvulsive SE, lymphocytic predominance in CSF were more commonly observed in the patients with autoimmune SE, while a high CSF total protein level, pleocytosis, and reduced glucose ratio were more common in those with infectious SE. EEG and MRI are important tools to confirm the diagnosis of SE and exclude structural lesions $(19,28)$, but were not particularly helpful in the current study.
TABLE 1 | Demographic data of inflammatory SE patients.

\begin{tabular}{|c|c|}
\hline & Patients ( $n=46$ ) \\
\hline Onset age (years) & $45(28-60)$ \\
\hline Female & $25(54.3)$ \\
\hline \multicolumn{2}{|l|}{ Onset symptom } \\
\hline Fever & $24(52.2)$ \\
\hline Decreased consciousness & $17(37.0)$ \\
\hline Seizure & $10(21.7)$ \\
\hline Upper respiratory tract infection & $8(17.4)$ \\
\hline Headache & $6(13.0)$ \\
\hline Psychosis & $6(13.0)$ \\
\hline Fatigue & $3(6.5)$ \\
\hline Cognitive decline & $1(2.2)$ \\
\hline Latency of seizure after onset symptoms (days) & $3(0-7)$ \\
\hline STESS $\geq 3$ at admission & $18(39.1)$ \\
\hline END-IT score $\geq 3$ at admission & $43(93.5)$ \\
\hline SE with prominent motor symptoms & $37(80.4)$ \\
\hline Generalized convulsive SE & $25(54.3)$ \\
\hline Epilepsia partialis continua & $6(13.0)$ \\
\hline Focal onset evolving into bilateral convulsive SE & $4(8.7)$ \\
\hline Myoclonic SE with coma & $1(2.2)$ \\
\hline Hyperkinetic SE & $1(2.2)$ \\
\hline Non-convulsive SE & 9 (19.6) \\
\hline Non-convulsive SE with coma & $6(13.0)$ \\
\hline Myoclonic absence status & $1(2.2)$ \\
\hline Non-convulsive SE without impairment of consciousness & $1(2.2)$ \\
\hline Aphasic status & $1(2.2)$ \\
\hline Number of AEDs used & $3(2-3)$ \\
\hline Refractory SE & 36 (78.3) \\
\hline Super refractory SE & $19(41.3)$ \\
\hline Required general anesthesia for SE control & $21(45.7)$ \\
\hline Death during admission & $13(28.3)$ \\
\hline Days of admission & $39(26-79)$ \\
\hline Days in ICU & $33.5(11-60)$ \\
\hline More than two AEDs at discharge & $17(36.9)$ \\
\hline Good outcome at discharge $(\mathrm{mRS}<3)$ & $17(36.9)$ \\
\hline Good outcome at last follow up (mRS<3) & $16(34.8)$ \\
\hline
\end{tabular}

Continuous variables were presented as median (interquartile range) Categorical variables were presented as $n(\%)$.

$A E D$, antiepileptic drug; ICU, intensive care unit; mRS, modified Rankin Scale; SE, status epilepticus; STESS, status epilepticus severity score.

Among all patients with SE, infection accounted for $4.2 \%$ and autoimmune accounted for $5 \%$. This suggests that autoimmune SE is as common as infectious SE $(20,29)$, and therefore clinical features that can distinguish the two entities are important for intensive care physicians who care for patients with SE. We observed some differences in the presenting features of those with autoimmune and infectious SE. The age at onset was younger in the patients with autoimmune SE, which has also been reported in two previous studies $(11,20)$. Female predominance was also observed in the autoimmune SE group in this study, which is in accordance with previous reports that reported females predominance in autoimmune encephalitis and systemic autoimmune disorders $(14,30,31)$. 
TABLE 2 | Comparison of the clinical features of autoimmune and infectious SE.

\begin{tabular}{|c|c|c|c|c|}
\hline & $\begin{array}{c}\text { Autoimmune } \\
\text { SE }(n=25)\end{array}$ & $\begin{array}{l}\text { Infectious SE } \\
(n=21)\end{array}$ & $p$-value & OR $(95 \% \mathrm{Cl})$ \\
\hline Onset age (years) & $32(23-49.5)$ & $56(36.5-68.5)$ & 0.015 & \\
\hline Female & $17(68.0)$ & $8(38.1)$ & 0.043 & $0.29(0.09-0.98)$ \\
\hline \multicolumn{5}{|l|}{ Onset symptom } \\
\hline Fever & $13(52.0)$ & $11(52.4)$ & 0.979 & $0.99(0.31-3.15)$ \\
\hline Decrease consciousness & $9(36.0)$ & $8(38.1)$ & 0.883 & $0.91(0.28-3.04)$ \\
\hline Seizure & $6(24.0)$ & $4(19.0)$ & 0.685 & $1.34(0.32-5.58)$ \\
\hline Upper respiratory tract infection & $5(20.0)$ & $3(14.3)$ & 0.611 & $1.50(0.31-7.19)$ \\
\hline Headache & $2(8.0)$ & $4(19.0)$ & 0.268 & $0.37(0.06-2.26)$ \\
\hline Psychosis & $6(24.0)$ & $0(0.0)$ & 0.025 & \\
\hline Fatigue & $1(4.0)$ & $2(9.5)$ & 0.450 & $0.4(0.03-4.70)$ \\
\hline Cognitive decline & $0(0.0)$ & $1(4.8)$ & 0.806 & $0.41(0.01-11.68)$ \\
\hline Latency of seizure after onset symptoms (days) & $3(0-7)$ & $2(0-8.5)$ & 0.892 & \\
\hline STESS $\geq 3$ at admission & $11(44.0)$ & 7 (33.3) & 0.460 & $1.57(0.47-5.23)$ \\
\hline END-IT score $\geq 3$ at admission & $23(92.0)$ & 20 (95.2) & 1.000 & $1.74(0.15-20.65)$ \\
\hline SE with prominent motor symptoms & $17(68.0)$ & $20(95.2)$ & 0.027 & $0.11(0.01-0.94)$ \\
\hline Generalized convulsive SE & 11 & 14 & & \\
\hline Epilepsia partialis continua & 3 & 3 & & \\
\hline Focal onset evolving into bilateral convulsive SE & 2 & 2 & & \\
\hline Myoclonic SE with coma & 0 & 1 & & \\
\hline Hyperkinetic SE & 1 & 0 & & \\
\hline Non-convulsive SE & $8(32.0)$ & $1(4.8)$ & 0.027 & $9.41(1.07-83.01)$ \\
\hline Non-convulsive SE with coma & 5 & 1 & & \\
\hline Myoclonic absence status & 1 & 0 & & \\
\hline Non-convulsive SE without impairment of consciousness & 1 & 0 & & \\
\hline Aphasic status & 1 & 0 & & \\
\hline Number of AED used & $3(2-3)$ & $3(1-3)$ & 0.159 & \\
\hline Refractory SE & $22(88.0)$ & $14(66.7)$ & 0.081 & $3.67(0.81-16.59)$ \\
\hline Super refractory SE & $15(41.3)$ & $4(19.0)$ & 0.007 & $6.38(1.65-24.63)$ \\
\hline Required general anesthesia for SE control & $16(64.0)$ & $5(23.8)$ & 0.006 & $5.69(1.56-20.76)$ \\
\hline Death during admission & $5(20.0)$ & $8(38.1)$ & 0.175 & $0.41(0.11-1.52)$ \\
\hline Days of admission & $40(21-91)$ & $33(26-77.5)$ & 0.817 & \\
\hline Days in ICU & $34(10.5-63.5)$ & $33(15-57)$ & 0.869 & \\
\hline More than two AEDs at discharge & $11(44.0)$ & $6(28.6)$ & 0.280 & $1.96(0.57-6.74)$ \\
\hline Good prognosis at discharge $(\mathrm{mRS}<3)$ & $11(44.0)$ & $6(28.6)$ & 0.280 & $1.96(0.57-6.74)$ \\
\hline Good prognosis at last follow up $(\mathrm{mRS}<3)$ & $10(66.7)$ & $6(50.0)$ & 0.381 & $2.00(0.42-9.52)$ \\
\hline
\end{tabular}

Continuous variables were presented as median (interquartile range).

Categorical variables were presented as $n(\%)$.

AED, antiepileptic drug; CI, confidence interval; ICU, intensive care unit; mRS, modified Rankin Scale; OR, odds ratio; SE, status epilepticus; STESS, status epilepticus severity score.

The onset symptoms of autoimmune SE can be various. Alteration in mental status is the cardinal symptom (12), but provides little information about the underlying etiology. In our patients, the presenting symptoms of inflammatory SE included fever, decreased consciousness, seizure, upper respiratory tract infection, headache, psychosis, fatigue, and cognitive decline. Of note, psychosis was present only in those with autoimmune SE and not in those with infectious SE. Other studies have also reported that psychosis is the dominant presenting symptom among patients with autoimmune encephalitis $(14,29,32,33)$. In addition, we found that more of the patients with autoimmune SE had non-convulsive SE compared to those with infectious SE, which was not reported in the two previous studies $(11,20)$. This may be due to the difficulty in recognizing non-convulsive
SE clinically without EEG monitoring or because it was not specifically looked for. Super refractory SE was also more prevalent in the autoimmune SE group, which may be due to the difficulty in making a diagnosis and the ineffectiveness of traditional SE treatment to control seizure activity without immunotherapy (34). When non-convulsive SE or psychosis followed by SE occurs in patients with a young age and female sex, autoimmune SE should be considered.

CSF studies are an important tool to identify the cause of SE, however, such studies can be challenging clinically. Neutrophilic predominant pleocytosis usually points toward a bacterial infection or the early stage of viral encephalitis, especially in the first 24 to 48 hours (24). Lymphocytic predominant pleocytosis was associated with autoimmune SE in our study, 
TABLE 3 | The findings of the first cerebrospinal fluid (CSF) and electroencephalography (EEG) studies.

\begin{tabular}{|c|c|c|c|c|}
\hline & $\begin{array}{l}\text { Autoimmune SE } \\
\quad(n=25)\end{array}$ & Infectious SE $(n=21)$ & $p$-value & OR (95\% Cl) \\
\hline \multicolumn{5}{|l|}{ The first CSF findings } \\
\hline CSF WBC count (cell/ $\left./ \mathrm{mm}^{3}\right)$ & $3(0-18)$ & $20(2.5-536)$ & 0.011 & \\
\hline Neutrophilic predominance & $2(15.4)$ & $9(52.9)$ & 0.034 & $0.162(0.03-0.96)$ \\
\hline Lymphocytic predominance & $11(84.6)$ & $8(47.1)$ & 0.034 & $6.19(1.04-36.78)$ \\
\hline${ }^{b} \lg G$ index $>0.6$ & $11(55.0)$ & $4(66.7)$ & 0.612 & $0.61(0.09-4.14)$ \\
\hline \multicolumn{5}{|l|}{ The first EEG finding } \\
\hline Normal & $1(4.0)$ & $3(14.3)$ & 0.318 & $0.25(0.02-2.61)$ \\
\hline Background slowing activity & $14(56.0)$ & $6(28.6)$ & 0.081 & $0.51(0.24-1.09)$ \\
\hline Sporadic epileptiform discharge & $2(8.0)$ & $4(19.0)$ & 0.239 & $2.38(0.48-11.74)$ \\
\hline Periodic discharge & $3(12.0)$ & $3(14.3)$ & 0.769 & $1.19(0.27-5.29)$ \\
\hline
\end{tabular}

Continuous variables were presented as median (interquartile range).

Categorical variables were presented as $n(\%)$.

Cl, confidence interval; OR, odds ratio; SE, status epilepticus; WBC, white blood cell.

a The CSF/blood glucose ratio was available in 16 patients with infectious and all patients with autoimmune etiology.

b The IgG index was available in six patients with infectious and 20 patients with autoimmune etiology.

but it was also often seen in cases of viral encephalitis-related SE $(17,24)$. Intensive care physicians often face a dilemma over whether to use antiviral therapy or immunotherapy when the diagnosis is unclear. Other parameters of the CSF can aid in the differential diagnosis, as our data suggested that the patients with an infectious etiology usually had a higher CSF protein level, although prolonged SE itself may result in a milder elevation of lactate and/or total protein levels. This was also reported by Oyanguren et al. who found similar white blood cell count between patients with viral infections and autoimmune processes, but that the protein level was higher in those with a CNS viral infection (35). Therefore, a high protein level in patients with lymphocytic predominance pleocytosis may suggest a viral etiology.

MRI can aid in the search for the etiology of SE, but with limitations. Limbic encephalitis may present as an increased FLAIR/T2 signal or abnormal DWI in the medial temporal lobes (36-38), and it can be used in helping to make the diagnosis of autoimmune encephalitis (16). Prolonged SE itself can also cause similar changes to some viral infectious in MRI signal with DWI abnormalities in the hippocampus and pulvinar (39), particularly herpes simplex encephalitis (18). Furthermore, these MRI patterns may not be present in all types of autoimmune SE and one study reported that $60 \%$ of the MRI findings in patients with anti-NMDA encephalitis may have been normal (14). Our data showed that no specific MRI findings could differentiate autoimmune and infectious SE.

EEG is routinely used to evaluate patients with seizures or disturbed consciousness. Slow background activity was more dominant in autoimmune patients compared with other etiologies of seizure (40), although we found no statistical difference in EEG findings between the two groups. Our study showed that at an early stage of inflammatory SE, it remains difficult to differentiate the two entities using currently available para-clinical investigations. The early use of auto-antibody assays may be needed when autoimmune SE is suspected clinically.

We found that general anesthesia was more commonly used in the patients with autoimmune SE. This is in accordance with previous studies in which patients with autoimmune SE were less responsive to $\operatorname{AEDs}(11,20,30,41)$. The reason why AEDs are less effective for autoimmune SE remains to be clarified, although it is well-known that the treatment of autoimmune SE requires prompt immunotherapy $(14,15)$, which may then reduce the use of general anesthesia.

The functional outcomes were similar in both infectious and autoimmune groups with a similar mRS score at discharge and similar mortality rate during admission. However, most of our patients had a poor outcome at discharge or last follow-up (63.0 and $65.2 \%$, respectively). Our study showed that the predictive values of STESS and END-IT scores were not in the same direction. That is, STESS was more sensitive but END-IT was more specific in terms of predicting the outcomes at discharge. More studies may be needed to compare the use of these two scores. In addition to functional impairments, a recent study reported that patients also had substantial impairments in their quality of life after SE (42). Our patients with autoimmune SE had a mortality rate of $20 \%$, which is similar to other studies ranging from 10 to $23 \%(20,30,43)$. A recent population-based study conducted in Germany reported a hospital mortality rate for all types of SE of $14.8 \%$ with a higher rate in those with refractory SE and super refractory SE (15.0 and 39.9\%, respectively) (44). The higher mortality rate in patients with autoimmune etiology compared to those with all-cause SE may be related to the high percentage of super refractory SE among patients with an autoimmune etiology. This higher mortality rate compared to all-cause SE emphasize the need for rapid recognition of the condition and prompt treatment toward the underlying causes in addition to standard SE care. 
TABLE 4 | The findings of magnetic resonance imaging study.

\begin{tabular}{|c|c|c|c|c|}
\hline & $\begin{array}{l}\text { Autoimmune } \\
\text { SE }(n=25)\end{array}$ & $\begin{array}{l}\text { Infectious } \\
\text { SE }(n=21)\end{array}$ & $p$-value & OR (95\% Cl) \\
\hline FLAIR and T2 abnormalities & $11(44)$ & $8(38.1)$ & 0.685 & $1.28(0.39-4.17)$ \\
\hline Unilateral & $5(20.0)$ & $3(14.3)$ & 0.729 & $0.72(0.11-4.62)$ \\
\hline Bilateral & $6(24.0)$ & $5(23.8)$ & & \\
\hline Mesial temporal lobe & $7(28)$ & $4(19)$ & & \\
\hline Lateral temporal lobe & $2(8)$ & $1(4.8)$ & & \\
\hline Frontal lobe & $6(24)$ & $3(14.3)$ & & \\
\hline Parietal lobe & $7(28)$ & $2(9.5)$ & & \\
\hline Occipital lobe & $7(28)$ & $4(19)$ & & \\
\hline Unilateral & $5(35.7)$ & $6(60)$ & 0.408 & $0.37(0.07-1.97)$ \\
\hline Bilateral & $9(64.3)$ & $4(40)$ & 0.408 & $2.7(0.51-14.37)$ \\
\hline \multicolumn{5}{|l|}{ Location } \\
\hline Temporal lobe & 11 (78.6) & $7(70)$ & & \\
\hline Mesial temporal lobe & $7(50)$ & $3(30)$ & & \\
\hline Lateral temporal lobe & $4(28.6)$ & $5(50)$ & & \\
\hline Frontal lobe & $6(42.9)$ & $5(50)$ & & \\
\hline Parietal lobe & $6(42.9)$ & $5(50)$ & & \\
\hline Occipital lobe & $5(35.7)$ & $5(50)$ & & \\
\hline Basal ganglion & $0(0)$ & $4(40)$ & & \\
\hline Multiple lobes & $11(78.6)$ & $6(60)$ & 0.393 & $2.44(0.41-14.75)$ \\
\hline
\end{tabular}

Categorical variables were presented as $n(\%)$.

Cl, confidence interval; DWI, diffusion-weighted imaging; FLAIR, fluid-attenuated inversion recovery; OR, odds ratio; SE, status epilepticus.

The limitations of this study are that it was conducted at a single hospital and that the design was retrospective. In addition, the study was started before the availability of recent autoimmune encephalitis screening tests and immunotherapies, which may have affected the outcomes.

In conclusion, we observed that patients with autoimmune SE had a younger age at onset, female predominance, and often presented with psychosis, super-refractory SE and nonconvulsive SE. The initial clinical investigations including EEG and MRI only provided limited information about the underlying etiology. CSF tests were helpful in diagnosing bacterial infectious-related SE but had difficulty in differentiating viral encephalitis and autoimmune SE. Since these two etiologies have different treatment strategies and the presenting symptoms are quite similar $(12,29)$, it is important to differentiate the two conditions as soon as possible. The patient characteristics and presenting features identified in our study may provide clinicians with some clues about the underlying etiology. Empiric treatment can be given based on these clinical clues while waiting for the results of more definitive diagnostic tests such as viral serology tests and neuronal surface auto-antibody screening.

\section{ETHICS STATEMENT}

This study is approved by the Chang Gung Medical Foundation Institutional Review Board (IRB No.: 103-3665B and 201800677B0).

\section{AUTHOR CONTRIBUTIONS}

All authors have read and approved the final manuscript. C-HL contributed to clinical data analysis and draft of the manuscript. Y-TL, C-JH, and F-YS had contributions to clinical data acquisition and analysis. M-HT had substantial contributions to the conception and design of the study, data analysis, critical revision, and final approval of the manuscript.

\section{FUNDING}

This work was supported in part by research grant CMRPG8G0131 to C-HL from Kaohsiung Chang Gung Memorial Hospital, Taiwan. 


\section{REFERENCES}

1. Bermeo-Ovalle A, Bleck T. Status epilepticus in the intensive care unit. Semin Neurol. (2016) 36:550-9. doi: 10.1055/s-0036-1592357

2. Wu YW, Shek DW, Garcia PA, Zhao S, Johnston SC. Incidence and mortality of generalized convulsive status epilepticus in California. Neurology (2002) 58:1070-6. doi: 10.1212/WNL.58.7.1070

3. Lv RJ, Wang Q, Cui T, Zhu F, Shao XQ. Status epilepticus-related etiology, incidence and mortality: a meta-analysis. Epilepsy Res. (2017) 136:12-7. doi: 10.1016/j.eplepsyres.2017.07.006

4. Glauser T, Shinnar S, Gloss D, Alldredge B, Arya R, Bainbridge $\mathrm{J}$, et al. Evidence-based guideline: treatment of convulsive status epilepticus in children and adults: report of the guideline committee of the American epilepsy society. Epilepsy Curr. (2016) 16:48-61. doi: 10.5698/1535-7597-16.1.48

5. Jagoda A, Riggio S. Refractory status epilepticus in adults. Ann Emerg Med. (1993) 22:1337-48. doi: 10.1016/S0196-0644(05)80120-9

6. Tsai MH, Chuang YC, Chang HW, Chang WN, Lai SL, Huang CR, et al. Factors predictive of outcome in patients with de novo status epilepticus. QJM (2009) 102:57-62. doi: 10.1093/qjmed/hcn149

7. Koubeissi M, Alshekhlee A. In-hospital mortality of generalized convulsive status epilepticus: a large US sample. Neurology (2007) 69:886-93. doi: 10.1212/01.wnl.0000269791.96189.70

8. Rossetti AO, Hurwitz S, Logroscino G, Bromfield EB. Prognosis of status epilepticus: role of aetiology, age, and consciousness impairment at presentation. J Neurol Neurosurg Psychiatry (2006) 77:611-5. doi: 10.1136/jnnp.2005.080887

9. Seinfeld S, Goodkin HP, Shinnar S. Status epilepticus. Cold Spring Harb perspect Med. (2016) 6:a022830. doi: 10.1101/cshperspect.a022830

10. Singh TD, Fugate JE, Rabinstein AA. The spectrum of acute encephalitis: causes, management, and predictors of outcome. Neurology (2015) 84:359-66. doi: 10.1212/WNL.0000000000001190

11. Shin JW, Koo YS, Kim YS, Kim DW, Kim KK, Lee SY, et al. Clinical characterization of unknown/cryptogenic status epilepticus suspected as encephalitis: a multicenter cohort study. J Neuroimmunol. (2018) 315:1-8. doi: 10.1016/j.jneuroim.2017.12.004

12. Venkatesan A, Tunkel AR, Bloch KC, Lauring AS, Sejvar J, Bitnun A, et al. Case definitions, diagnostic algorithms, and priorities in encephalitis: consensus statement of the international encephalitis consortium. Clin Infect Dis. (2013) 57:1114-28. doi: 10.1093/cid/cit458

13. Suthar R, Sankhyan N. Bacterial Infections of the central nervous system. Indian J Pediatr. (2018) 86:60-9. doi: 10.1007/s12098-017-2477-Z

14. Titulaer MJ, McCracken L, Gabilondo I, Armangue T, Glaser C, Iizuka T, et al. Treatment and prognostic factors for long-term outcome in patients with antiNMDA receptor encephalitis: an observational cohort study. Lancet Neurol. (2013) 12:157-65. doi: 10.1016/S1474-4422(12)70310-1

15. Shin YW, Lee ST, Park KI, Jung KH, Jung KY, Lee SK, et al. Treatment strategies for autoimmune encephalitis. Ther Adv Neurol Disord. (2018) 11:1756285617722347. doi: 10.1177/1756285617722347

16. Graus F, Titulaer MJ, Balu R, Benseler S, Bien CG, Cellucci T, et al. A clinical approach to diagnosis of autoimmune encephalitis. Lancet Neurol. (2016) 15:391-404. doi: 10.1016/S1474-4422(15)00401-9

17. Armangue T, Leypoldt F, Dalmau J. Autoimmune encephalitis as differential diagnosis of infectious encephalitis. Curr Opin Neurol. (2014) 27:361-8. doi: 10.1097/WCO.0000000000000087

18. Sureka J, Jakkani RK. Clinico-radiological spectrum of bilateral temporal lobe hyperintensity: a retrospective review. Br J Radiol. (2012) 85:e782-92. doi: $10.1259 / \mathrm{bjr} / 30039090$

19. Lancaster E. The diagnosis and treatment of autoimmune encephalitis. J Clin Neurol. (2016) 12:1-13. doi: 10.3988/jcn.2016.12.1.1

20. Spatola M, Novy J, Du Pasquier R, Dalmau J, Rossetti AO. Status epilepticus of inflammatory etiology: a cohort study. Neurology (2015) 85:464-70. doi: 10.1212/WNL.0000000000001717

21. Brophy GM, Bell R, Claassen J, Alldredge B, Bleck TP, Glauser $\mathrm{T}$, et al. Guidelines for the evaluation and management of status epilepticus. Neurocrit Care (2012) 17:3-23. doi: 10.1007/s12028-0129695-z
22. Ferlisi M, Shorvon S. The outcome of therapies in refractory and superrefractory convulsive status epilepticus and recommendations for therapy. Brain (2012) 135(Pt 8):2314-28. doi: 10.1093/brain/aws091

23. Trinka E, Cock H, Hesdorffer D, Rossetti AO, Scheffer IE, Shinnar S, et al. A definition and classification of status epilepticus-Report of the ILAE task force on classification of status epilepticus. Epilepsia (2015) 56:1515-23. doi: 10.1111/epi.13121

24. Tuppeny M. Viral meningitis and encephalitis. Crit Care Nurs Clin North Am. (2013) 25:363-80. doi: 10.1016/j.ccell.2013.04.003

25. Rossetti AO, Logroscino G, Milligan TA, Michaelides C, Ruffieux C, Bromfield EB. Status Epilepticus Severity Score (STESS): a tool to orient early treatment strategy. J Neurol. (2008) 255:1561-6. doi: 10.1007/s00415-008-0989-1

26. Gao Q, Ou-Yang TP, Sun XL, Yang F, Wu C, Kang T, et al. Prediction of functional outcome in patients with convulsive status epilepticus: the END-IT score. Crit Care (2016) 20:46. doi: 10.1186/s13054-016-1221-9

27. Hirsch LJ, LaRoche SM, Gaspard N, Gerard E, Svoronos A, Herman ST, et al. American clinical neurophysiology society's standardized critical care EEG terminology: 2012 version. J Clin Neurophysiol. (2013) 30:1-27. doi: 10.1097/WNP.0b013e3182784729

28. Heine J, Pruss H, Bartsch T, Ploner CJ, Paul F, Finke C. Imaging of autoimmune encephalitis-Relevance for clinical practice and hippocampal function. Neuroscience (2015) 309:68-83. doi: 10.1016/j.neuroscience.2015.05.037

29. Saraya A, Mahavihakanont A, Shuangshoti S, Sittidetboripat N, Deesudchit $\mathrm{T}$, Callahan $\mathrm{M}$, et al. Autoimmune causes of encephalitis syndrome in Thailand: prospective study of 103 patients. BMC Neurol. (2013) 13:150. doi: 10.1186/1471-2377-13-150

30. Holzer FJ, Rossetti AO, Heritier-Barras AC, Zumsteg D, Roebling R, Huber R, et al. Antibody-mediated status epilepticus: a retrospective multicenter survey. Eur Neurol. (2012) 68:310-7. doi: 10.1159/000341143

31. Gubbels Bupp MR, Potluri T, Fink AL, Klein SL. The confluence of sex hormones and aging on immunity. Front Immunol. (2018) 9:1269. doi: 10.3389/fimmu.2018.01269

32. Hao Q, Wang D, Guo L, Zhang B. Clinical characterization of autoimmune encephalitis and psychosis. Compr Psychiatry (2017) 74:9-14. doi: 10.1016/j.comppsych.2016.12.006

33. Lim JA, Lee ST, Jung KH, Kim S, Shin JW, Moon J, et al. Anti-N-methyl$\mathrm{d}$-aspartate receptor encephalitis in Korea: clinical features, treatment, and outcome. J Clin Neurol. (2014) 10:157-61. doi: 10.3988/jcn.2014.10.2.157

34. Varley J, Taylor J, Irani SR. Autoantibody-mediated diseases of the CNS: structure, dysfunction and therapy. Neuropharmacology (2018) 132:71-82. doi: 10.1016/j.neuropharm.2017.04.046

35. Oyanguren B, Sanchez V, Gonzalez FJ, de Felipe A, Esteban L, Lopez-Sendon JL, et al. Limbic encephalitis: a clinical-radiological comparison between herpetic and autoimmune etiologies. Eur J Neurol. (2013) 20:1566-70. doi: 10.1111/ene.12249

36. Lai M, Hughes EG, Peng X, Zhou L, Gleichman AJ, Shu H, et al. AMPA receptor antibodies in limbic encephalitis alter synaptic receptor location. Ann Neurol. (2009) 65:424-34. doi: 10.1002/ana.21589

37. Lai M, Huijbers MG, Lancaster E, Graus F, Bataller L, Balice-Gordon R, et al. Investigation of LGI1 as the antigen in limbic encephalitis previously attributed to potassium channels: a case series. Lancet Neurol. (2010) 9:776-85. doi: 10.1016/S1474-4422(10)70137-X

38. Hoftberger R, Titulaer MJ, Sabater L, Dome B, Rozsas A, Hegedus B, et al. Encephalitis and GABAB receptor antibodies: novel findings in a new case series of 20 patients. Neurology (2013) 81:1500-6. doi: 10.1212/WNL.0b013e3182a9585f

39. Mendes A, Sampaio L. Brain magnetic resonance in status epilepticus: a focused review. Seizure (2016) 38:63-7. doi: 10.1016/j.seizure.2016.04.007

40. Limotai C, Denlertchaikul C, Saraya AW, Jirasakuldej S. Predictive values and specificity of electroencephalographic findings in autoimmune encephalitis diagnosis. Epilepsy Behav. (2018) 84:29-36. doi: 10.1016/j.yebeh.2018.04.007

41. Dubey D, Blackburn K, Greenberg B, Stuve O, Vernino S. Diagnostic and therapeutic strategies for management of autoimmune encephalopathies. Expert Rev Neurother. (2016) 16:937-49. doi: 10.1080/14737175.2016.1189328

42. Kortland LM, Knake S, von Podewils F, Rosenow F, Strzelczyk A. Socioeconomic outcome and quality of life in adults after status epilepticus: a 
multicenter, longitudinal, matched case-control analysis from Germany. Front Neurol. (2017) 8:507. doi: 10.3389/fneur.2017.00507

43. Holzer FJ, Seeck M, Korff CM. Autoimmunity and inflammation in status epilepticus: from concepts to therapies. Expert Rev Neurother. (2014) 14:1181-202. doi: 10.1586/14737175.2014. 956457

44. Strzelczyk A, Ansorge S, Hapfelmeier J, Bonthapally V, Erder $\mathrm{MH}$, Rosenow F. Costs, length of stay, and mortality of super-refractory status epilepticus: a population-based study from Germany. Epilepsia (2017) 58:1533-41. doi: 10.1111/epi. 13837
Conflict of Interest Statement: The authors declare that the research was conducted in the absence of any commercial or financial relationships that could be construed as a potential conflict of interest.

Copyright (c) 2019 Lin, Lu, Ho, Shih and Tsai. This is an open-access article distributed under the terms of the Creative Commons Attribution License (CC BY). The use, distribution or reproduction in other forums is permitted, provided the original author(s) and the copyright owner(s) are credited and that the original publication in this journal is cited, in accordance with accepted academic practice. No use, distribution or reproduction is permitted which does not comply with these terms. 